\title{
Programa educativo: la salud bucal como parte de una vida saludable (Parte II)
}

\section{Educational program: oral health as part of healthy life (Part II)}

\author{
Moreno $\mathrm{MA}^{1}$, Arena $\mathrm{AL}^{2}$, Gallardo $\mathrm{ME}^{2}$, Alloatti MB${ }^{1}$ \\ ${ }^{1}$ Universidad Nacional de Córdoba, Facultad de Odontología, Cátedra de Periodoncia "B" \\ ${ }^{2}$ Universidad Nacional de Córdoba, Facultad de Odontología, Cátedra de Endoncia "B"
}

\begin{abstract}
Oral diseases are often overlooked as a source of infection with the potential to compromise the general health. Health finds its place in the school environment par excellence, so it was intended, through this project, to complement the development of different curricular disciplines in order to share, meet needs and interests and build knowledge by providing a means to promote health at School level. Objectives: Through an inter and multidisciplinary team, formed by university professors of basic and clinical subjects of the Faculty of Dentistry of the National University of Córdoba (UNC) Argentina, it was intended to bring to the school environment the scientific knowledge linked to health promotion and prevention. These teachers acted as training agents for students from two secondary institutions located in the province of Córdoba, with the aim of training agents of change and promoters of oral health. Methods: The activities were developed in stages, a first of Action Design, a second of Execution of the activity, a third of material preparation and a fourth of oral health promotion. Results: 51 students were incorporated as health promoters, 86 attended the dentist, 355 students received Education for Health and 6 teachers were trained. Conclusion: Education is an appropriate instrument to generate habits in favor of a healthy life. Teamwork, interaction, construction and appropriation of knowledge linked to the promotion and prevention of oral health are fundamental axes not only for the achievement of immediate results but also to generate a multiplier effect that lasts over time.
\end{abstract}

KEY WORDS: oral health, students, promotion, quality of life, training.

\section{Resumen}

Las enfermedades orales a menudo son pasadas por alto como fuente de infección con potencial de comprometer la salud general del individuo. La salud encuentra en el ámbito escolar su escenario por excelencia, por lo que se pretendió mediante este proyecto complementar y acompañar el desarrollo de distintas disciplinas curriculares con la finalidad de compartir, conocer necesidades e intereses y construir conocimientos proporcionando un arma que permita la promoción a nivel escolar.

Objetivo: Por medio de un equipo inter y multidisciplinario, conformado por docentes universitarios de asignaturas básicas y clínicas de la Facultad de Odontología de la Universidad Nacional de Córdoba (UNC) Argentina, se pretendió llevar al ámbito escolar el conocimiento científico vinculado a la promoción de salud y prevención. Estos docentes actuaron como agentes capacitadores de alumnos de dos instituciones secundarias ubicadas en la provincia de Córdoba, con el propósito de formar agentes de cambio y promotores de la salud bucodental. Métodos: Las actividades se desarrollaron por etapas, una primera de Diseño de acción, una segunda de Ejecución de la actividad, una tercera de confección de material y una cuarta de promoción de salud bucal. Resultados: Se incorporaron 51 alumnos como promotores de salud, 86 al examen bucal, 355 alumnos recibieron Educación para la Salud y se capacitaron 6 docentes. Conclusión: La educación constituye una herramienta apropiada para generar hábitos a favor de una vida saludable. El trabajo en equipo, la interacción, la construcción y apropiación de conocimientos vinculados a la promoción y prevención en salud bucal son ejes fundamentales no sólo para el logro de resultados inmediatos sino para generar un efecto multiplicador que perdure en el tiempo.

PALABRAS CLAVE: salud bucal, estudiantes, promoción, calidad de vida, capacitación. 


\section{Introducción}

Las enfermedades orales como posibles fuentes de infección con capacidad de comprometer la salud general de la población son a menudo desestimadas por ellos, en general por falta de información ${ }^{1}$.

El concepto de calidad de vida relacionado a salud oral acuñado en los últimos años requiere considerar, no sólo factores tales como el malestar, dolor o alteraciones de la función, sino también debe incluir aspectos emocionales y funciones sociales asociadas a la salud oral ${ }^{2,3}$.

Frente a la problemática situación estructural de exclusión económica, social y educativa, se propuso el desarrollo de diferentes estrategias y ámbitos de trabajo para llevar el conocimiento científico al ámbito escolar, estimulando la investigación, expresión e intercambio entre alumnos, educadores y comunidad.

La salud encuentra en el sistema escolar su escenario por excelencia. Es por ello que se propuso el desarrollo de un programa de educación-investigación-acción integrado en Instituciones de nivel medio de la ciudad de Córdoba.

Por medio de un equipo inter y multidisciplinario, conformado por docentes universitarios de asignaturas básicas y clínicas de la Facultad de Odontología de la UNC, se pretendió llevar al ámbito escolar el conocimiento científico vinculando promoción de salud y prevención. Los docentes actuaron como agentes capacitadores, con el objetivo de formar agentes de cambio y promotores de la salud buco-dental.

A partir de este proyecto se pretendió proporcionar a los adolescentes los conocimientos necesarios para la promoción y protección de la salud individual, familiar y de la comunidad; fomentando la responsabilidad y el trabajo grupal organizado mediante el uso de las destrezas habituales y capacidades características de esta etapa de vida ${ }^{4}$

Procuramos llevar el conocimiento científico a los adolescentes, buscando nuevas formas de estar y de aprender en la escuela. Revalorizar, rescatar, fortalecer y crear espacios que permitieran construir una estrategia educativa, promoviendo la participación de los jóvenes en diferentes acciones organizadas a favor de una vida saludable, dirigidas especialmente a valorar la importancia de la prevención de la salud bucal, como factor de riesgo en la salud integral del individuo ${ }^{5}$.

\section{Métodos}

El programa fue llevado a cabo en los Institutos secundarios Nuestra Señora del Sagrado Corazón (INSSC), ubicado en Av. Revolución de Mayo 1476 y Jóvenes Argentinos (JA), ubicado en Av. Ricchieri 3400. Fue dirigido a directivos, docentes y alumnos de 4to, 5to y 6to año con orientación a Ciencias Naturales.

El equipo de trabajo estuvo constituido por no docentes y docentes universitarios de asignaturas básicas y clínicas de la Facultad de Odontología de la UNC.

Las actividades se desarrollaron por etapas:

\section{Primera etapa: Diseño de acción}

En primera instancia se realizó en ambos colegios una reunión de acercamiento y consenso con la comunidad educativa, como espacio de capacitación, discusión y reflexión conjunta. Se presentaron a los integrantes del grupo de trabajo, se expuso el proyecto y se evaluó su grado de compromiso por participar. Se entregaron cuestionarios a docentes y alumnos que no habían participado en el proyecto anterior, con el objetivo de recoger datos relacionados a sus creencias y/o sus conocimientos referentes a la problemática planteada. Luego se realizó una segunda reunión como espacio de trabajo, intercambio y construcción conjunta de ejes temáticos. Según necesidades e intereses de docentes y alumnos se implementaron talleres-debate en los cursos de 4 to, 5to y 6to.

\section{Segunda etapa: Ejecución de la actividad}

Los talleres consistieron en espacios de intercambio y reflexión conjunta entre docentes de diferentes cátedras de la facultad y docentes y estudiantes de la institución.

En el INSSC se realizó en 4to año un encuentro modalidad taller "Caries dental" y 1 (una) demostración práctica en el laboratorio de la institución sobre "Importancia de las Células epiteliales de la cavidad bucal como mecanismo de defensa" (Fig. 1A). En 6to año se realizaron 3 (tres) encuentros modalidad taller, "Enfermedad periodontal y su repercusión a nivel sistémico"; "Cáncer bucal: importancia del control de las mucosas, lesiones pre-cancerosas, manifestaciones bucales de enfermedades de transmisión sexual" y "Traumatismo dentario: cómo actuar ante el mismo". Se realizó un encuentro debate "Enfermedades de transmisión sexual" y "Automedicación, alcohol y drogas" 
con la participación de 200 alumnos de 4to, 5to y 6to año (Fig. 1B).

En el Instituto JA se realizaron en 4to año 4 (cuatro) encuentros, "La salud bucal como parte de la salud general del individuo"; "Traumatismo dentario: cómo actuar ante el mismo"; "Saliva, su importancia en el mecanismo de defensa del organismo y en la transmisión de enfermedades" (Fig. 1C); "Técnicas de higiene oral" y 1 (una) demostración práctica en el laboratorio de la institución sobre "Importancia de las Células epiteliales de la cavidad bucal como mecanismo de defensa". En 5to año se trabajó principalmente con el espacio escolar de Proyectos de Investigación en el cual los alumnos propusieron realizar investigaciones de tipo epidemiológicas con respecto a las metas para la salud bucal del año 2000 (dos mil) y otros estándares de salud bucal (OMS 2012). En 6to año se realizaron 6 (seis) talleres debate, "Enfermedad periodontal y su repercusión a nivel sistémico"; "Caries dental. Teórico y muestra de un video sobre el proceso de desmineralización del diente"; "Cáncer bucal: importancia del control de las mucosas, lesiones pre cancerosas, manifestaciones bucales de enfermedades de transmisión sexual." A pedido de los docentes se incorporaron a este taller los alumnos de 4to año; "Enfermedades de transmisión sexual"; "Automedicación, alcohol y drogas y "Técnicas de higiene oral".

\section{Tercera etapa: Confección del material}

Con la intención de difundir el conocimiento y promover la salud bucal como parte de una vida saludable se propuso la elaboración de material didáctico mediante el uso de estrategias: obras de teatro, títeres, folletos, videos, posters, recursos en internet; todas acciones organizadas y ejecutadas por adolescentes voluntarios, acompañados por docentes de diferentes espacios curriculares y supervisados por integrantes de nuestro equipo.

\section{Cuarta etapa: Promoción de salud bucal}

En el JA en 4to año el proyecto fue el incentivo que culminó con la preparación de actividades de promoción de la salud bucal para ejecutar tanto dentro de la escuela como en otras escuelas de la zona, en el marco del espacio de opción institucional: Educación para la Salud y la materia Formación para la Vida y el Trabajo. Los alumnos elaboraron 6 proyectos de intervención comunitaria en promoción de la salud bucal, que fueron puestos en marcha en la escuela primaria 20 de Junio ubicada en Bv. del Comercio 835, cada uno respectivamente de $1^{\circ}$ a $6^{\circ}$ grado. Los mismos presentaron actividades adecuadas a cada edad (títeres, cuentos, juegos didácticos, etc.) (Fig. $1 \mathrm{D}$ ).

Alumnos de 5to año plantearon una investigación acerca de los conocimientos sobre gingivitis de individuos de Córdoba, su prevención e incidencia, que será llevada a cabo el próximo año. En 6to año se iniciaron una serie de investigaciones acerca de distintas temáticas como hábitos higiénicos de individuos de Córdoba, epidemiología en Córdoba, nivel de conocimiento de individuos en temas básicos de salud bucal, cumplimiento con "Metas mínimas de salud bucal a alcanzar para el año 2000" y otros índices publicado por la OMS.

En el INSSC 10 (diez) alumnas de 6to año apoyadas por integrantes del equipo y docentes fueron las encargadas de confeccionar posters sobre prevención de enfermedades de salud bucal y técnicas de higiene que fueron colocados en el colegio, folletos para entregar a alumnos y padres y material didáctico que fue subido a la página web del colegio.

Todos los años un grupo de alumnos, profesores y hermanas del INSSC realizan un viaje a un colegio en Santa Victoria Este (salta) con la misión de evangelizar en la fe y transmitir conocimientos para mejorar la calidad de vida de esta comunidad. Allí asiste gente del lugar y poblaciones vecinas con muchas necesidades insatisfechas y falta de posibilidades. Se propuso llevar conocimientos relacionados a promoción y prevención de salud a la comunidad, por lo que se elaboraron folletos, juegos, charlas debate, material didáctico y se capacitó a los promotores de salud en prevención de enfermedades bucales y enseñanzas de técnicas de higiene. (Figuras 1 E-F). 


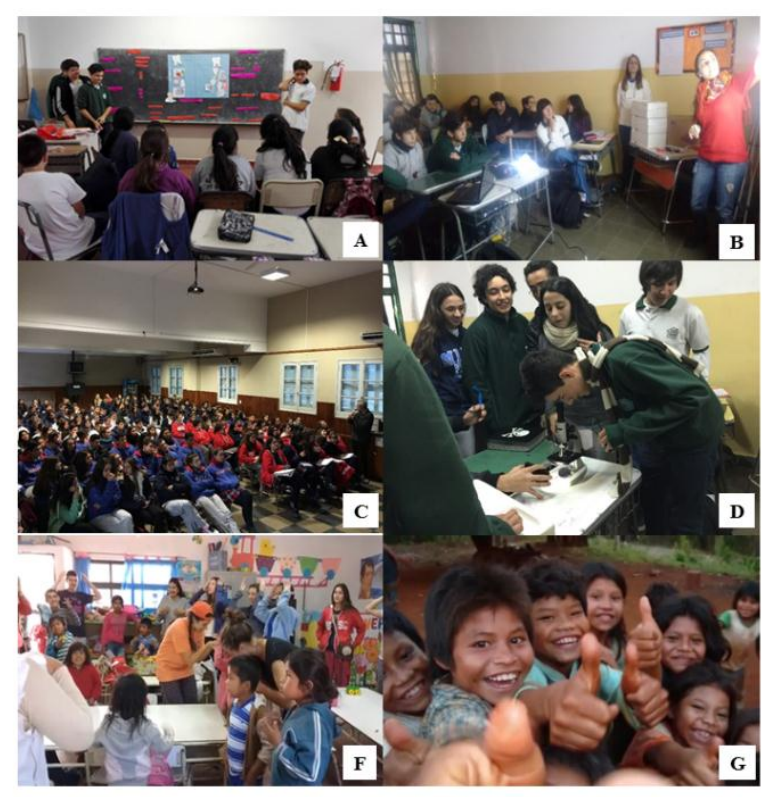

Figura 1 A-F: Distintos momentos de la actividad extensionista

\section{Resultados}

En el instituto Nuestra Señora del Sagrado Corazón se realizaron 2 (dos) charlas debate en 4to año donde se pudo capacitar a un total de 37 alumnos y 3 (tres) charlas debate en 6to donde se capacitó a un total de 28 alumnos. Además se realizó una charla debate sobre "Enfermedades de transmisión sexual" "Automediación, alcohol y drogas" donde se capacitaron a 200 (doscientos) alumnos, con la participaron de 6 (seis) docentes y el sacerdote de la institución. Los docentes capacitados y que participaron en la ejecución del proyecto fueron 3 (tres). Los alumnos voluntarios que se incorporaron como promotores juveniles fueron 10 (diez), quienes confeccionaron material didáctico sobre temas que consideraron de mayor interés, coordinados por becarias y docentes. Los adolescentes que concurrieron al odontólogo a lo largo del año fueron un total de 35 alumnos.

En el Instituto Jóvenes Argentinos se realizaron 5 charlas debate en 4to año donde se capacitó a un total de 39 alumnos y 5 charlas debate en 6to año donde se capacitaron a 26 (veintiséis) alumnos.

Los docentes capacitados y que participaron en la ejecución del proyecto fueron 3 (tres).

Los alumnos voluntarios que se incorporaron como promotores juveniles fueron 31 (treintaiuno) , quienes concurrieron a la escuela 20 de junio donde organizaron y realizaron 6 (seis) charlas debate distribuidas en los 6 (seis) grados primarios donde se capacitó a un total de 98 (noventaiocho) alumnos.
Los adolescentes que concurrieron al odontólogo a lo largo del año fueron un total de 28 alumnos.

\section{Discusión y Conclusión}

Sobre la base de los resultados obtenidos podemos concluir

a) La educación constituye una herramienta apropiada para generar hábitos a favor de una vida saludable.

b) El trabajo en equipo, la interacción, la construcción y apropiación de conocimientos vinculados a la promoción y prevención en salud bucal son ejes fundamentales no sólo para el logro de resultados inmediatos sino para que el efecto multiplicador perdure en el tiempo.

c) La propuesta extensionista se articuló perfectamente con el proyecto curricular de la escuela para su Ciclo Orientado en Ciencias Naturales, ya que el mismo intentó formar promotores de salud e investigadores científicos escolares (perfil del egresado), permitiendo el debate y la planificación de diferentes proyectos por parte de los alumnos coordinados por sus docentes.

\section{Recomendaciones}

- Apoyar e incentivar la incorporación de programas de prevención de salud bucal en el ámbito escolar, concientizando a docentes y alumnos sobre la importancia de la salud oral como parte de la salud general, logrando la extensión de los conocimientos a la comunidad.

- Asumir un compromiso como agentes de cambio y promotores de salud, transmitiendo ejes valiosos y relevantes a ser considerados para la elección de un hacer y un ser como parte de un proyecto de vida.

\section{Agradecimientos}

$\mathrm{Al}$ equipo de trabajo formado por: V. Huespe Rico; S. Soto; A. Azcurra; R. Panico; C. Francia; P. Verducci; M. Usin; M. Caravajal; C. Sakalian; A. Moyano; C. Calafell; V. Doro.

Al personal directivo, profesores y alumnos del INSSC y Jóvenes Argentinos. Este proyecto fue aprobado y avalado por la Secretaria de Extensión de la Universidad Nacional de Córdoba (Res. HC Superior Nº1279/16). 
Todos los autores declaran que no existen conflictos potenciales de interés con respecto a la autoría y/o publicación de este artículo.

All authors declare no potential conflicts of interest with respect to the authorship and/or publication of this article.

\section{Referencias}

1. Casey Hey. El papel del profesional en la educación del público sobre la importancia de la Salud Oral. Enfermedad Periodontal y Salud General: Una Guía para el Clínico. Copyright, 2011 por la Compañía ColgatePalmolive.

2. Trinchitella AB. Importancia de la salud oral y su conexión con la salud. Biomedicina, 2006, 2 (3) - 246251

3. Meneses Gómez EJ. Salud bucodental y calidad de vida oral en los mayores. 2010. Tesis doctoral. Universidad Complutense de Madrid. Facultad de Odontología. Departamento de Estomatología IV (Profilaxis, Odontopediatría y Ortodoncia) Disponible en [http://eprints.ucm.es/12303/1/T32521.pdf] Consultado $10 / 6 / 2016$

4. Zurro AM, Jodar Solá G (2011) Atención primaria de salud y atención familiar y comunitaria. España: Elsevier S.L, 2011;1-16.

5. Cançado M, Faustino-Silva D, Lansing J. El papel del odontólogo como facilitador de la relación con el paciente adolescente. Revisión de literatura Odontoestomatología. 12(14):16-28 Montevideo 2010. [Consultado: 28/05/15] Disponible en: http://www.lume. ufrgs.br/bitstream/handle/10183/108122/000754499.pdf ?sequence $=111$

Correspondencia a / Corresponding to: Dra. Mirta Analía Moreno Facultad de Odontología UNC-Córdoba Argentina. Correo electrónico (e-mail): mirtacalafell@gmail.com 